\title{
SPECTRA OF CONSTRUCTS OF A SYSTEM OF OPERATORS
}

\author{
ANGEL CARRILLO AND CARLOS HERNÁNDEZ
}

\begin{abstract}
This paper describes the spectrum and the upper and lower Fredholm spectra of $(n+m)$-tuples $\left(F\left(A_{1}\right), \ldots, F\left(A_{n}\right), G\left(B_{1}\right), \ldots, G\left(B_{m}\right)\right)$ of operators, where $\left(A_{i}\right)$ and $\left(B_{j}\right)$ are systems of operators in two Hilbert spaces $\mathcal{H}_{1}$ and $\mathcal{H}_{2}$, and $F$ and $G$ are certain linear operators defined on $\mathcal{L}\left(\mathcal{H}_{i}\right)$. Using spectral mapping theorems the spectra of operators constructed by the action of a polynomial on a system $\left(F\left(A_{1}\right), \ldots, F\left(A_{n}\right), G\left(B_{1}\right), \ldots, G\left(B_{m}\right)\right)$ is obtained. In particular, the spectra of the elementary operator and tensor products of operators is determined.
\end{abstract}

1. Introduction. During the last years there has been progress in calculating the spectrum and the essential spectrum of various operators formed from others. Thus the spectrum $\omega$, where $\omega=\sigma_{l}, \sigma_{r}, \sigma_{e}^{+}$or $\sigma_{e}^{-}$, has been determined for the tensor product $A \otimes B$ of two operators in a Hilbert space $[2,8, \mathbf{1 1}]$, the generalized derivation $\tau_{A B}(X)=A X-X B[\mathbf{5 , 8}]$ and the multiplication operator $S_{A B}(X)=$ $A X B[6,8]$.

For the elementary operator $W(X)=\sum_{i=1}^{n} A_{i} X B_{i}$, Lumer and Rosenblum [10] and Fialkow [6] proved some partial results about $\sigma(W)$ and $\sigma_{e}(W)$, respectively. On the other hand, Curto [4] obtained $\sigma(W)$ in terms of the Taylor spectra of $\left(A_{i}\right)$ and $\left(B_{i}\right)$.

In [8] Robin Harte determined the spectra of certain operators, in particular $A \otimes B, S_{A B}$ and $\tau_{A B}$, using the concept of joint spectrum, introduced in [7], and spectral mapping theorems $[\mathbf{7 , 8}]$. In this note, following that line, and stressing the algebraic properties of the maps $A \rightarrow A \otimes I$, and $R_{B}\left(=S_{I B}\right)$, we calculate the spectrum $\omega$ of polynomial constructs of some kind of systems of operators. From this calculation we set down explicit representation for $\omega(W)$ and in the case where $\left(A_{i}\right)$ and $\left(B_{i}\right)$ are commutative systems of operators in Hilbert spaces we recover all the results mentioned above, with the exception of the one due to Curto, but we use it to show that

$$
\sigma(W)=\sigma\left(\left(A_{i}\right)\right) \circ \sigma\left(\left(B_{i}\right)\right)=\sigma_{T}\left(\left(A_{i}\right)\right) \circ \sigma_{T}\left(\left(B_{i}\right)\right) .
$$

Let us introduce some notation and recall some relevant definitions.

Let $B$ be a complex Banach space, $\mathcal{L}(B)$ the algebra of all linear bounded operators and $n$ a natural number. The operators Column $(\mathrm{Col})$ and Row are defined

Received by the editors February 8, 1983 and, in revised form, September 14, 1983. Some results of this paper were presented in 6 e Congrès du Groupement des Mathématiciens d'Expression Latine, Luxembourg 1981.

1980 Mathematics Subject Classification. Primary 47A10, 47A53, 47A25; Secondary 46M05.

Key words and phrases. Spectrum, joint spectrum, upper and lower Fredholm spectra, essential spectrum, Fredholm operator, spectral mapping theorem, elementary operator, tensor products. 
from $\mathcal{L}(B)^{n}$ into $\mathcal{L}\left(B, B^{n}\right)$ and $\mathcal{L}\left(B^{n}, B\right)$, respectively, by the following rules:

$$
\begin{aligned}
& \operatorname{Col}\left(T_{1}, \ldots, T_{n}\right)(x)=\left(T_{1} x, \ldots, T_{n} x\right), \\
& \operatorname{Row}\left(T_{1}, \ldots, T_{n}\right)\left(x_{1}, \ldots, x_{n}\right)=T_{1} x_{1}+\cdots+T_{n} x_{n} .
\end{aligned}
$$

$\mathcal{L}(B)^{n}$ is a linear space under the usual operations. For any two elements $S=$ $\left(S_{1}, \ldots, S_{n}\right)$ and $T=\left(T_{1}, \ldots, T_{n}\right)$ in this space, define

$$
S \circ T=\operatorname{Row}\left(S_{1}, \ldots, S_{n}\right) \operatorname{Col}\left(T_{1}, \ldots, T_{n}\right)=S_{1} T_{1}+\cdots+S_{n} T_{n} .
$$

(If $s, t \in \mathbf{C}^{n}$, define $s \circ t=s_{1} t_{1}+\cdots+s_{n} t_{n}$.)

Let $B_{1}$ be a complex Banach space and $F: B_{1} \rightarrow B$ a linear operator. $F^{n}$ will denote the function

$$
F^{n}\left(y_{1}, \ldots, y_{n}\right)=\left(F\left(y_{1}\right), \ldots, F\left(y_{n}\right)\right) .
$$

The left, right, upper Fredholm and lower Fredholm spectra of a system $T=$ $\left(T_{1}, \ldots, T_{n}\right)$ of operators in $B$ are defined $[\mathbf{1}, \mathbf{7}]$ as

$$
\begin{aligned}
& \sigma_{l}(T)=\left\{s \in \mathbf{C}^{n}: \operatorname{Col}(T-s) \text { is not left invertible }\right\}, \\
& \sigma_{r}(T)=\left\{s \in \mathbf{C}^{n}: \operatorname{Row}(T-s) \text { is not right invertible }\right\}, \\
& \sigma_{e}^{+}(T)=\left\{s \in \mathbf{C}^{n}: \operatorname{Col}(T-s) \text { is not upper Fredholm }\right\}, \\
& \sigma_{e}^{-}(T)=\left\{s \in \mathbf{C}^{n}: \operatorname{Row}(T-s) \text { is not lower Fredholm }\right\} .
\end{aligned}
$$

The union $\sigma(T)=\sigma_{l}(T) \cup \sigma_{r}(T)$ will be called the joint spectrum (or simply spectrum) of $T$. In case $n=1$ the union $\sigma_{e}^{+}(T) \cup \sigma_{e}^{-}(T)$ is the Wolf essential spectrum $\left(\sigma_{e}(T)\right)$ of $T$ which coincides with $\sigma(\pi(T))$, where $\pi$ is the canonical homomorphism of $\mathcal{L}(B)$ onto the Calkin algebra over $B$. Moreover, if $n=1$ and $B$ is a Hilbert space, then $\sigma_{e}^{+}(T)$ and $\sigma_{e}^{-}(T)$ are the left and right essential spectra of $T[\mathbf{1 2}]$.

Throughout the note $\mathcal{H}_{i}(i=1,2)$ and $\mathcal{Y}$ will denote a complex, separable Hilbert space and a complex Banach space, respectively. All the spaces are assumed to be infinite dimensional and if $x \in \mathscr{H}_{1}$ and $y \in \mathscr{H}_{2}$ then $x \otimes y$ will denote the operator defined by $(x \otimes y)(z)=\langle z, y\rangle x$.

2. The spectra of $\left(F\left(A_{1}\right), \ldots, F\left(A_{n}\right), G\left(B_{1}\right), \ldots, G\left(B_{m}\right)\right)$. The functions $F(A)=A \otimes I$ and $G(B)=I \otimes B$ are homomorphisms (of algebras and continuous) from $\mathcal{L}\left(\mathscr{H}_{1}\right)$ and $\mathcal{L}\left(\mathscr{H}_{2}\right)$ to $\mathcal{L}\left(\mathscr{H}_{1} \otimes \mathscr{H}_{2}\right)$, respectively, and $A \otimes B=F(A) G(B)$.

On the other hand, if $L_{A}$ and $R_{B}$ are the multiplication operators $L_{A}(X)=$ $A X$ and $R_{B}(X)=X B$, then $F(A)=L_{A}$ is a homomorphism from $\mathcal{L}\left(\mathscr{H}_{1}\right)$ to $\mathcal{L}\left(\mathcal{L}\left(\mathcal{H}_{2}, \mathscr{H}_{1}\right)\right), G(B)=R_{B}$ is an antihomomorphism (i.e. a bounded linear operator such that $\left.G\left(B B^{\prime}\right)=G\left(B^{\prime}\right) G(B)\right)$ from $\mathcal{L}\left(\mathcal{H}_{2}\right)$ to $\mathcal{L}\left(\mathcal{L}\left(\mathcal{H}_{2}, \mathscr{H}_{1}\right)\right)$ and $W=$ $\sum_{i=1}^{n} F\left(A_{i}\right) G\left(B_{i}\right)$.

It follows that the particular operators considered in the introduction can be constructed by the action of a polynomial on a system

$$
\left(F\left(A_{1}\right), \ldots, F\left(A_{n}\right), G\left(B_{1}\right), \ldots, G\left(B_{m}\right)\right)
$$

of operators, where $F$ is a homomorphism and $G$ is a homomorphism or an antihomomorphism. So the spectral mapping theorems enable us to determine the spectra of those operators by the calculation of the spectra of that kind of system. 
THEOREM 1. Let $F$ be a homomorphism from $\mathcal{L}\left(\mathcal{H}_{1}\right)$ into $\mathcal{L}(\mathcal{Y})$, and $G$ a homomorphism or antihomomorphism from $\mathcal{L}\left(\mathcal{H}_{2}\right)$ into $\mathcal{L}(\mathcal{Y})$. If $\mathcal{F}=F^{n}$ and $\mathcal{G}=G^{m}$, then, for any pair of systems $A=\left(A_{1}, \ldots, A_{n}\right)$ and $B=\left(B_{1}, \ldots, B_{m}\right)$ of bounded linear operators in $\mathfrak{H}_{1}$ and $\mathfrak{H}_{2}$, respectively, we have

$$
\begin{gathered}
\sigma_{l}(\mathcal{F}(A), \mathcal{G}(B)) \subset \sigma_{l}(A) \times \sigma_{l}(B) \quad \text { if } G \text { is a homomorphism, } \\
\sigma_{l}(\mathcal{F}(A), \mathcal{G}(B)) \subset \sigma_{l}(A) \times \sigma_{r}(B) \quad \text { if } G \text { is an antihomomorphism. }
\end{gathered}
$$

Moreover, if

$$
F(x \otimes x) G(y \otimes y)=G(y \otimes y) F(x \otimes x) \not \equiv 0
$$

for each $(x, y) \in \mathscr{H}_{1} \times \mathscr{H}_{2}$ with $x \neq 0$ and $y \neq 0$, then

$$
\begin{gathered}
\sigma_{l}(\mathcal{F}(A), \mathcal{G}(B))=\sigma_{l}(A) \times \sigma_{l}(B) \quad \text { if } G \text { is a homomorphism, } \\
\sigma_{l}(\mathcal{F}(A), \mathcal{G}(B))=\sigma_{l}(A) \times \sigma_{r}(B) \quad \text { if } G \text { is an antihomomorphism. }
\end{gathered}
$$

Proof. (We shall only prove (1.2) and (1.4).) Let $(s, t)=\left(s_{1}, \ldots, s_{n}, t_{1}, \ldots\right.$, $\left.t_{m}\right)$ be in $\mathbf{C}^{n+m}$. If $(s, t) \notin \sigma_{l}(A) \times \sigma_{r}(B)$, then there exists a system $C=$ $\left(C_{1}, \ldots, C_{n}\right)$ of operators in $\mathfrak{H}_{2}$ such that $\mathcal{F}(C) \circ \mathcal{F}(A-s)=I$ or there exists a system $D=\left(D_{1}, \ldots, D_{m}\right)$ of operators in $\mathfrak{H}_{2}$ for which $\mathcal{G}(D) \circ \mathcal{G}(B-t)=I$. Therefore, $T \circ(\mathcal{F}(A-s), \mathcal{G}(B-t))=I$, with $T=\left(F\left(C_{1}\right), \ldots, F\left(C_{n}\right), 0, \ldots, 0\right)$ or $T=\left(0, \ldots, 0, G\left(D_{1}\right), \ldots, G\left(D_{m}\right)\right)$. In other words $(s, t) \notin \sigma_{l}(\mathcal{F}(A), \mathcal{G}(B))$ and (1.2) has been established.

In order to prove (1.4), suppose $(s, t) \in \sigma_{l}(A) \times \sigma_{r}(B)$. The operators $L=$ $\operatorname{Col}(A-s)$ and $R^{*}=\operatorname{Col}\left(B_{1}^{*}-\bar{t}_{1}, \ldots, B_{m}^{*}-\bar{t}_{m}\right)$ are not left invertible. There exist two normal sequences $\left(x_{k}\right)$ and $\left(y_{k}\right)$ in $\mathscr{H}_{1}$ and $\mathscr{H}_{2}$, respectively, such that $L\left(x_{k}\right) \rightarrow 0$ and $R^{*}\left(y_{k}\right) \rightarrow 0$.

Let $T_{k}=F\left(x_{k} \otimes x_{k}\right) G\left(y_{k} \otimes y_{k}\right)$ for $k \geq 1$. By hypothesis $T_{k} \not \equiv 0$ and

$$
F\left(x_{k} \otimes x_{k}\right) T_{k}=G\left(y_{k} \otimes y_{k}\right) T_{k}=T_{k} .
$$

For $k \geq 1$, choose $z_{k} \in \operatorname{Ran} T_{k}$ with $\left\|z_{k}\right\|=1$. The element $z_{k}$ is a fixed point of $F\left(x_{k} \otimes x_{k}\right)$ and $G\left(y_{k} \otimes y_{k}\right)$. Then,

$$
\begin{aligned}
\left\|F\left(A_{i}-s_{i}\right)\left(z_{k}\right)\right\| & =\left\|F\left(A_{i}-s_{i}\right) F\left(x_{k} \otimes x_{k}\right)\left(z_{k}\right)\right\| \\
& \leq\|F\|\left\|\left(A_{i}-s_{i}\right) x_{k}\right\|\left\|x_{k}\right\|\left\|z_{k}\right\|=\|F\|\left\|\left(A_{i}-s_{i}\right) x_{k}\right\|
\end{aligned}
$$

for all $k \geq 1$ and $1 \leq i \leq n$. Thus, $\lim _{k \rightarrow \infty} F\left(A_{i}-s_{i}\right)\left(z_{k}\right)=0$ for $1 \leq i \leq n$. Similarly, $\lim _{k \rightarrow \infty} G\left(B_{j}-t_{j}\right)\left(z_{k}\right)=0$ for $1 \leq j \leq m$.

Therefore, $\operatorname{Col}(\mathcal{F}(A-s), \mathcal{G}(B-t))\left(z_{k}\right) \rightarrow 0$, when $k \rightarrow \infty$, and so $(s, t) \in$ $\sigma_{l}(\mathcal{F}(A), \mathcal{G}(B))$.

By reversing products in $\mathcal{L}(\mathcal{Y}), \mathcal{L}\left(\mathscr{H}_{1}\right)$ and $\mathcal{L}\left(\mathscr{H}_{2}\right)$ we obtain, under the hypothesis of the theorem, the following relations:

$$
\begin{aligned}
& \sigma_{r}(\mathcal{F}(A), \mathcal{G}(B)) \subset \sigma_{r}(A) \times \sigma_{r}(B), \\
& \sigma_{r}(\mathcal{F}(A), \mathcal{G}(B)) \subset \sigma_{r}(A) \times \sigma_{l}(B), \\
& \sigma_{r}(\mathcal{F}(A), \mathcal{G}(B))=\sigma_{r}(A) \times \sigma_{r}(B), \\
& \sigma_{r}(\mathcal{F}(A), \mathcal{G}(B))=\sigma_{r}(A) \times \sigma_{l}(B) .
\end{aligned}
$$


THEOREM 2. Let $A, B, \mathcal{F}$ and $\mathcal{G}$ be as before.

(a) Suppose that $F\left(K_{1}\right) G\left(K_{2}\right)$ is a compact operator whenever $K_{1}$ and $K_{2}$ are compact, then

$$
\begin{aligned}
& \sigma_{e}^{+}(\mathcal{F}(A), \mathcal{G}(B)) \subset\left(\sigma_{e}^{+}(A) \times \sigma_{l}(B)\right) \cup\left(\sigma_{l}(A) \times \sigma_{e}^{+}(B)\right), \\
& \sigma_{e}^{-}(\mathcal{F}(A), \mathcal{G}(B)) \subset\left(\sigma_{e}^{-}(A) \times \sigma_{r}(B)\right) \cup\left(\sigma_{r}(A) \times \sigma_{e}^{-}(B)\right)
\end{aligned}
$$

if $G$ is a homomorphism;

$$
\begin{aligned}
& \sigma_{e}^{+}(\mathcal{F}(A), \mathcal{G}(B)) \subset\left(\sigma_{e}^{+}(A) \times \sigma_{r}(B)\right) \cup\left(\sigma_{l}(A) \times \sigma_{e}^{-}(B)\right), \\
& \sigma_{e}^{-}(\mathcal{F}(A), \mathcal{G}(B)) \subset\left(\sigma_{e}^{-}(A) \times \sigma_{l}(B)\right) \cup\left(\sigma_{r}(A) \times \sigma_{e}^{+}(B)\right)
\end{aligned}
$$

if $G$ is an antihomomorphism.

(b) If $F(x \otimes x) G(y \otimes y)=G(y \otimes y) F(x \otimes x) \not \equiv 0$ for all $(x, y) \in \mathscr{H}_{1} \times \mathscr{H}_{2}$ with $x \neq 0$ and $y \neq 0$, then

$$
\begin{aligned}
& \sigma_{e}^{+}(\mathcal{F}(A), \mathcal{G}(B)) \supset\left(\sigma_{e}^{+}(A) \times \sigma_{l}(B)\right) \cup\left(\sigma_{l}(A) \times \sigma_{e}^{+}(B)\right), \\
& \sigma_{e}^{-}(\mathcal{F}(A), \mathcal{G}(B)) \supset\left(\sigma_{e}^{-}(A) \times \sigma_{r}(B)\right) \cup\left(\sigma_{r}(A) \times \sigma_{e}^{-}(B)\right)
\end{aligned}
$$

if $G$ is a homomorphism;

$$
\begin{aligned}
& \sigma_{e}^{+}(\mathcal{F}(A), \mathcal{G}(B)) \supset\left(\sigma_{e}^{+}(A) \times \sigma_{r}(B)\right) \cup\left(\sigma_{l}(A) \times \sigma_{e}^{-}(B)\right), \\
& \sigma_{e}^{-}(\mathcal{F}(A), \mathcal{G}(B)) \supset\left(\sigma_{e}^{-}(A) \times \sigma_{l}(B)\right) \cup\left(\sigma_{r}(A) \times \sigma_{e}^{+}(B)\right)
\end{aligned}
$$

if $G$ is an antihomomorphism.

ProOF. We shall first prove (2.2). Let $(s, t)=\left(s_{1}, \ldots, s_{n}, t_{1}, \ldots, t_{m}\right) \in \mathbf{C}^{n+m}$. Suppose that $(s, t)$ is not in the right side of $(2.2)$. Thus, $(s, t) \notin \sigma_{l}(A) \times \sigma_{r}(B)$ or $(s, t) \in\left(\sigma_{l}(A) \backslash \sigma_{e}^{+}(A)\right) \times\left(\sigma_{r}(B) \backslash \sigma_{e}^{-}(B)\right)$. In the first case, by the preceding theorem, $(s, t) \notin \sigma_{l}(\mathcal{F}(A), \mathcal{G}(B))$, and so $(s, t) \notin \sigma_{e}^{+}(\mathcal{F}(A), \mathcal{G}(B))$. In the second one, there exist $C \in \mathcal{L}\left(\mathscr{H}_{1}\right)^{n}, D \in \mathcal{L}\left(\mathscr{H}_{2}\right)^{m}$ and two compact operators $K_{1}$ and $K_{2}$ in $\mathscr{H}_{1}$ and $\mathscr{H}_{2}$, respectively, such that

$$
C \circ(A-s)+K_{1}=I \quad \text { and } \quad(B-t) \circ D+K_{2}=I
$$

therefore,

$$
\left(\mathcal{F}(C), F\left(K_{1}\right) \mathcal{G}(D)\right) \circ(\mathcal{F}(A-s), \mathcal{G}(B-t))+F\left(K_{1}\right) G\left(K_{2}\right)=I
$$

where $F\left(K_{1}\right) \mathcal{G}(D)=\left(F\left(K_{1}\right) G\left(D_{1}\right), \ldots, F\left(K_{1}\right) G\left(D_{m}\right)\right)$. Then, in this case, we also have $(s, t) \notin \sigma_{e}^{+}(\mathcal{F}(A), \mathcal{G}(B))$.

To prove (2.4), choose $(s, t) \in \sigma_{e}^{+}(A) \times \sigma_{r}(B)$ and follow the proof of (1.4). The sequence $\left(x_{k}\right)$ can be assumed orthonormal and so the following additional property holds:

$$
F\left(x_{k} \otimes x_{k}\right) z_{k^{\prime}}=\left\{\begin{array}{cl}
0 & \text { if } k^{\prime} \neq k, \\
z_{k} & \text { if } k^{\prime}=k .
\end{array}\right.
$$

It can be proved that $\left\{z_{k}\right\}$ is a linear independent set. Let $\left(z_{k}^{\prime}\right)$ be a subsequence of $\left(z_{k}\right)$ without any convergent subsequence. We have

$$
\operatorname{Col}(\mathcal{F}(A-s), \mathcal{G}(B-t))\left(z_{k}^{\prime}\right) \rightarrow 0 \quad \text { when } k \rightarrow \infty
$$


because the same is true for $\left(z_{k}\right)$. Therefore, $(s, t) \in \sigma_{e}^{+}(\mathcal{F}(A), \mathcal{G}(B))$ and so $\sigma_{e}^{+}(A) \times \sigma_{r}(B) \subset \sigma_{e}^{+}(\mathcal{F}(A), \mathcal{G}(B))$.

The inclusion $\sigma_{1}(A) \times \sigma_{e}^{-}(B) \subset \sigma_{e}^{+}(\mathcal{F}(A), \mathcal{G}(B))$ can be proved in a similar way.

Finally, in order to prove (2.4.1), choose $(s, t) \in \sigma_{e}^{-}(A) \times \sigma_{1}(B)$, and write $(A-s)^{*}=\left(\left(A_{1}-s_{1}\right)^{*}, \ldots,\left(A_{n}-s_{n}\right)^{*}\right)$. Since $\operatorname{Col}\left((A-s)^{*}\right)$ is not upper Fredholm and $\operatorname{Col}(B-t)$ is not left invertible, it follows that there exist an orthonormal sequence $\left(x_{k}\right)$ and a normal sequence $\left(y_{k}\right)$ such that

$$
\operatorname{Col}\left((A-s)^{*}\right)\left(x_{k}\right) \rightarrow 0 \quad \text { and } \operatorname{Col}(B-t)\left(y_{k}\right) \rightarrow 0 .
$$

Let $T_{k}=F\left(x_{k} \otimes x_{k}\right) G\left(y_{k} \otimes y_{k}\right)$ for $k \geq 1$. For each $k \geq 1$, choose $z_{k} \in \operatorname{Ran} T_{k}$ with $\left\|z_{k}\right\|=1$ and take $U_{k} \in \mathcal{Y}^{\prime}$ such that $\left\|U_{k}\right\|=1$ and $U_{k}\left(z_{k}\right)=1$. If $V_{k}=U_{k} T_{k}$, then $V_{k}\left(z_{k}\right)=1,\left\|V_{k}\right\| \geq 1$ and $V_{k}=V_{k} F\left(x_{k} \otimes x_{k}\right)=V_{k} G\left(y_{k} \otimes y_{k}\right)$. Writing $V_{k}^{\prime}=V_{k} /\left\|V_{k}\right\|$ we have

$$
\begin{aligned}
\left\|\left(F\left(A_{i}-s_{i}\right)\right)^{*}\left(V_{k}^{\prime}\right)\right\| & =\left\|V_{k}^{\prime} F\left(A_{i}-s_{i}\right)\right\|=\left\|V_{k}^{\prime} F\left(x_{k} \otimes x_{k}\right) F\left(A_{i}-s_{i}\right)\right\| \\
& =\left\|V_{k}^{\prime} F\left(x_{k} \otimes\left(A_{i}-s_{i}\right)^{*} x_{k}\right)\right\| \leq\|F\|\left\|x_{k}\right\|\left\|\left(A_{i}-s_{i}\right)^{*} x_{k}\right\|
\end{aligned}
$$

for all $k \geq 1$ and $1 \leq i \leq n$.

On the other hand,

$$
\begin{aligned}
\left\|G\left(B_{j}-t_{j}\right)^{*} V_{k}^{\prime}\right\| & =\left\|V_{k}^{\prime} G\left(B_{j}-t_{j}\right)\right\|=\left\|V_{k}^{\prime} G\left(y_{k} \otimes y_{k}\right) G\left(B_{j}-t_{j}\right)\right\| \\
& =\left\|V_{k}^{\prime} G\left(\left(B_{j}-t_{j}\right)\left(y_{k} \otimes y_{k}\right)\right)\right\| \leq\|G\|\left\|\left(B_{j}-t_{j}\right)\left(y_{k}\right)\right\|
\end{aligned}
$$

for all $k \geq 1$ and $1 \leq j \leq m$.

Thus,

$$
\operatorname{Col}\left(\left(F\left(A_{1}-s_{1}\right)\right)^{*}, \ldots,\left(F\left(A_{n}-s_{n}\right)\right)^{*},\left(G\left(B_{1}-t_{1}\right)\right)^{*}, \ldots,\left(G\left(B_{m}-t_{m}\right)\right)^{*}\right)\left(V_{k}^{\prime}\right)
$$

converges to zero. Since

$$
V_{r}\left(z_{k}\right)= \begin{cases}0 & \text { if } r \neq k \\ 1 & \text { if } r=k\end{cases}
$$

it follows that $\left(V_{k}^{\prime}\right)$ has a subsequence without any convergent subsequence. Therefore,

$$
\operatorname{Col}\left(\left(F\left(A_{1}-s_{1}\right)\right)^{*}, \ldots,\left(F\left(A_{n}-s_{n}\right)\right)^{*},\left(G\left(B_{1}-t_{1}\right)\right)^{*}, \ldots,\left(G\left(B_{m}-t_{m}\right)\right)^{*}\right)
$$

is not upper Fredholm and so $(s, t) \in \sigma_{e}^{-}(\mathcal{F}(A), \mathcal{G}(B))$.

The inclusion $\sigma_{r}(A) \times \sigma_{e}^{+}(B) \subset \sigma_{e}^{-}(\mathcal{F}(A), \mathcal{G}(B))$ can be proved in a similar way.

An analogous reasoning leads us to (2.1), (2.3) and (2.3.1). The inclusions (2.1.1) and (2.2.1) follow by reversing products.

3. Applications. Combining the two theorems we can obtain the sipectrum $\omega$, where $\omega=\sigma_{e}, \sigma_{r}, \sigma_{e}^{+}$or $\sigma_{e}^{-}$, of $\mathcal{P}(\mathcal{F}(A), \mathcal{G}(B))$ when the system $(\mathcal{F}(\Lambda), \mathcal{G}(B))$ is commutative and $P$ is any system of polynomials from $\mathbf{C}^{n+m}$ to) $\mathbf{C}^{r}$ (spectiral mapping theorems). For example, we can state the following results concerning left and upper spectra.

COROllary 1. Let $A=\left(A_{1}, \ldots, A_{n}\right)$ and $B=\left(B_{1}, \ldots, B_{n}\right)$ be two commutative systems of bounded linear operators in $\mathcal{H}_{1}$ and $\mathcal{H}_{2}$, respectively. Let $W: \mathcal{L}\left(\mathcal{H}_{2}, \mathcal{H}_{1}\right)$ $\rightarrow \mathcal{L}\left(\mathcal{H}_{2}, \mathcal{H}_{1}\right)$ be the elementary operator $W(X)=\sum_{i=1}^{n} A_{i} X B_{i}$. Then

$$
\sigma_{l}(W)=\sigma_{l}(A) \circ \sigma_{r}(B),
$$




$$
\sigma_{e}^{+}(W)=\left(\sigma_{e}^{+}(A) \circ \sigma_{r}(B)\right) \cup\left(\sigma_{l}(A) \circ \sigma_{e}^{-}(B)\right) .
$$

ProOF. Let $F: \mathcal{L}\left(\mathscr{H}_{1}\right) \rightarrow \mathcal{L}\left(\mathcal{L}\left(\mathscr{H}_{2}, \mathscr{H}_{1}\right)\right)$ and $G: \mathcal{L}\left(\mathscr{H}_{2}\right) \rightarrow \mathcal{L}\left(\mathcal{L}\left(\mathscr{H}_{2}, \mathscr{H}_{1}\right)\right)$ be the operators defined by $F(A)=L_{A}$ and $G(B)=R_{B}$. It is clear that $F(A) G(B)=$ $G(B) F(A)$ for every $A$ and $B$. If $x_{i}$ and $y_{i}(i=1,2)$ are nonzero elements then $F\left(x_{1} \otimes x_{2}\right) G\left(y_{1} \otimes y_{2}\right)$ is a rank one operator; in fact its range is generated by $x_{1} \otimes y_{2}$. Thus $F\left(S_{1}\right) G\left(S_{2}\right)$ is of finite rank if $S_{1}$ and $S_{2}$ are of finite rank and therefore, $F\left(K_{1}\right) G\left(K_{2}\right)$ is a compact operator whenever $K_{1}$ and $K_{2}$ are compact.

If $\mathcal{P}$ is the polynomial given by

$$
\mathcal{P}\left(x_{1}, \ldots, x_{n}, y_{1}, \ldots, y_{n}\right)=\left(x_{1}, \ldots, x_{n}\right) \circ\left(y_{1}, \ldots, y_{n}\right)
$$

then, $W=\mathcal{P}(\mathcal{F}(A), \mathcal{G}(B))$. Therefore,

$$
\sigma_{l}(W)=\mathcal{P}\left(\sigma_{l}\left(L_{A_{1}}, \ldots, L_{A_{n}}, R_{B_{1}}, \ldots, R_{B_{n}}\right)=\mathcal{P}\left(\sigma_{l}(A) \times \sigma_{r}(B)\right)\right.
$$

and so $\sigma_{l}(W)=\sigma_{l}(A) \circ \sigma_{r}(B)$.

Similarly we establish (3.2).

Note. The joint spectrum is a subset of Taylor spectrum. Then, from (3.1), the corresponding expression for $\sigma_{r}(W)$ and Curto's result [4], we have $\sigma(W)=$ $\sigma(A) \circ \sigma(B)=\sigma_{T}(A) \circ \sigma_{T}(B)$.

When $A$ and $B$ have only one component or when more information about the systems $A$ and $B$ is available, then it is possible to express $\omega(W)$ in terms of the spectra of the operators $A_{i}$ and $B_{i}$.

COROLLARY $2[6]$.

$$
\begin{gathered}
\sigma_{l}\left(S_{A_{1}, B_{1}}\right)=\sigma_{l}\left(A_{1}\right) \sigma_{r}\left(B_{1}\right), \\
\sigma_{e}^{+}\left(S_{A_{1}, B_{1}}\right)=\sigma_{e}^{+}\left(A_{1}\right) \sigma_{r}\left(B_{1}\right) \cup \sigma_{l}\left(A_{1}\right) \sigma_{e}^{-}\left(B_{1}\right) .
\end{gathered}
$$

COROLLARY $3[\mathbf{5}]$.

$$
\begin{gathered}
\sigma_{l}\left(\tau_{A_{1}, B_{1}}\right)=\sigma_{l}\left(A_{1}\right)-\sigma_{r}\left(B_{1}\right), \\
\sigma_{e}^{+}\left(\tau_{A_{1}, B_{1}}\right)=\left(\sigma_{e}^{+}\left(A_{1}\right)-\sigma_{r}\left(B_{1}\right)\right) \cup\left(\sigma_{l}\left(A_{1}\right)-\sigma_{e}^{-}\left(B_{1}\right)\right) .
\end{gathered}
$$

Proof. Apply Corollary 1 to $\left(A_{1}, I\right)$ and $\left(I,-B_{1}\right)$ using the formulas

$$
\omega\left(A_{1}, I\right)=\omega\left(A_{1}\right) \times\{1\} \text { and } \omega(I,-B)=\{1\} \times \omega(-B) .
$$

Corollary 4 [10]. Let $A \in \mathcal{L}\left(\mathcal{H}_{1}\right)$ and $B \in \mathcal{L}\left(\mathscr{H}_{2}\right)$. Suppose that $f_{i}(\lambda)$ and $g_{i}(\lambda), i=1, \ldots, n$, are holomorphic in domains containing $\sigma(A)$ and $\sigma(B)$, respectively. If $W(X)=\sum_{i=1}^{n} f_{i}(A) X g_{i}(B)$ then

$$
\sigma_{l}(W)=\left\{\left(f_{i}(u)\right) \circ\left(g_{i}(v)\right): u \in \sigma_{l}(A) \text { and } v \in \sigma_{r}(B)\right\}
$$

and

$$
\sigma_{e}^{+}(W)=\left\{\left(f_{i}(u)\right) \circ\left(g_{i}(v)\right):(u, v) \in\left(\sigma_{l}(A) \times \sigma_{e}^{-}(B)\right) \cup\left(\sigma_{e}^{+}(A) \times \sigma_{r}(B)\right)\right\} .
$$

PROOF. The result follows from Corollary 1 and the equality

$$
\omega\left(f_{1}(A), \ldots, f_{n}(A)\right)=\left\{\left(f_{1}(u), \ldots, f_{n}(u)\right): u \in \omega(A)\right\} .
$$

CoRollary $5[\mathbf{2}, \mathbf{1 1}]$. Let $A_{1} \in \mathcal{L}\left(\mathscr{H}_{1}\right)$ and $B_{1} \in \mathcal{L}\left(\mathcal{H}_{2}\right)$,

$$
\sigma_{l}\left(A_{1} \otimes B_{1}\right)=\sigma_{l}\left(A_{1}\right) \sigma_{l}\left(B_{1}\right),
$$

$$
\sigma_{e}^{+}\left(A_{1} \otimes B_{1}\right)=\sigma_{e}^{+}\left(A_{1}\right) \sigma_{l}\left(B_{1}\right) \cup \sigma_{l}\left(A_{1}\right) \sigma_{e}^{+}\left(B_{1}\right) .
$$

PROOF. Let $F_{i}: \mathcal{L}\left(H_{i}\right) \rightarrow \mathcal{L}\left(\mathscr{H}_{1} \otimes \mathcal{H}_{2}\right), 1 \leq i \leq 2$, be the homomorphisms given by $F_{1}(A)=A \otimes I$, and $F_{2}(B)=I \otimes B$. We can obtain the result following the proof of Corollary 1 . 


\section{REFERENCES}

1. J. Buoni, R. Harte and T. Wickstead, Upper and lower Fredholm spectra, Proc. Amer. Math. Soc. 66 (1977), 309-314.

2. A. Brown and C. Pearcy, Spectra of tensor products of operators, Proc. Amer. Math. Soc. 17 (1966), 162-169.

3. S. R. Caradus, W. E. Pfaffenberger and B. Yood, Calkin algebras and algebras of operators on Banach spaces, Lecture Notes in Pure and Appl. Math., Vol. 9, Dekker, New York, 1974.

4. R. Curto, The spectrum of elementary operators, Abstracts Amer. Math. Soc. 2 (1981), 552.

5. L. A. Fialkow, Elements of spectral theory for generalized derivations. II, Canad. J. Math. 33 (1981), 1205-1231.

6. __ Essential spectra of elementary operators, Trans. Amer. Math. Soc. 267 (1981), 157-173.

7. R. Harte, Spectral mapping theorems, Proc. Roy. Irish. Acad. Sect. A 72 (1972), 89-107.

8. __ Spectral mapping theorems on a tensor product, Bull. Amer. Math. Soc. 79 (1973), 367-372.

9. T. Kato, Perturbation theory for linear operators, Springer-Verlag, Berlin, Heidelberg and New York, 1976.

10. G. Lumer and M. Rosenblum, Linear operator equations, Proc. Amer. Math. Soc. 10 (1959), 32-41.

11. E. de Oteyza, Sobre la figura espectral del producto tensorial de dos operadores, M. S. Thesis, Facultad de Ciencias, Universidad Nacional Autonoma de Mexico, 1980.

12. C. Pearcy, Some recent developments in operator theory, CBMS Regional Conf. Ser. in Math., no. 36, Amer. Math. Soc., 1978.

Instituto de Matematicas, Universidad NaCional Autonoma de MeXico, CiUDAD UNIVERSITARIA, MEXICO, D.F. 04510 